\title{
New pattern clinical study of adverse drug reaction to ART may lead to chase drug discovery in anti-retroviral drug towards zero adverse drug reaction
}

Rahul Hajare

Hajare R. New pattern clinical study of adverse drug reaction to ART may lead to chase drug discovery in anti-retroviral drug towards zero adverse drug reaction. Curr Res Integr Med 2017;2 (2)14-16.

ART drug is widely used Anti-HIV agents. Of these, molecular recognition in their molecular nucleus likes diarylpyrimidine, benzoxazinone, heterocyclic most present in ART. Apart from these, some hetero atom along with some special core group have been reported. They are effective and not very costly, though there are also possibilities of ART adverse reactions common with use of ART. Cross reactivity between for example Etravirine and Rilpivirine, earlier reported may exceed $30 \%$. Common adverse drug reaction for highly protein bound ART, includes hypersensitivity reactions were characterized by rash, fever and sometimes organ involvement including, but not limited to, severe rash or rash accompanied by fever, general malaise, fatigue, muscle or joint aches, blisters, oral lesions, conjunctivitis, hepatitis and eosinophilia. indices were included. Adverse drug reactions caused by drugs belonging to no longer their therapeutic effect or alter their adverse events profile. Hence, this study has undertaken to promote health care setting, so researcher movement initially began inside hospitals and clinics. This study adverse drug reaction has setting drug discovery in HIV technology in collaboration with microbiologist, physicians, pharmacist, nurse practitioners and other healthcare professionals then there is adverse reactions could have been prevented or may lead to zero adverse reaction ART. Also, this study has shown of critical analyzing the clinical profile of these reactions, grading them and trying to figure out ways to deliver actual drug discovery to prevent these adverse reactions. Reports were messaged to see clinical pharmacist stating explicitly that the clinical pharmacist cares for patients in all health care settings emphasizes two points: that clinical pharmacist provides care to their patients and that this practice can occur in any practice setting. The study aims to assess the role of clinical pharmacist in identification and reporting of adverse drug reactions in antiretroviral therapy and consideration of new pattern clinical study.

Key Words: HIV technology, adverse drug reaction, anti-retroviral therapy
$T_{i}$ $31^{\text {st }}$ UNAIDS Programme Coordinating Board (PCB) meeting took place in Geneva from 11-13 December 2012. There were more than 700000 less new HIV infections estimated globally in 2011 than in 2001. The road from 2.5 million new HIV infections in 2011 to zero new HIV infections is a long one and significant efforts are required to accelerate HIV prevention programmes. Sustained investments for access to antiretroviral therapy by donors and national governments have led to record numbers of lives being saved in the past six years. In 2011 more than half a million fewer people died from AIDS-related illnesses than six years earlier. It's a dramatic turning point. In 14 countries, AIDS-related deaths dropped by more than $50 \%$ between 2005 and 2011. Numbers can quantify, but alone cannot express the impact of each averted death on the whole community, including its children. The number of people dying from AIDS-related causes began to decline in the mid-2000s because of scaled up antiretroviral therapy and the steady decline in HIV incidence since the peak of the epidemic in 1997. In 2011, this decline continued, with evidence showing that the drop in the number of people dying from AIDS-related causes is accelerating in several countries. We report here novel combinatory clinical study of adverse drug reaction to ART. It has inconsistent development and single max variable result with combinations will lead to inclination to chase drug discovery.

\section{METHODS}

\section{Objectives}

This article provides an overview of adverse drug reaction to ART and its use in the management of drug discovery trials.

\section{Data sources}

A literature search of PubMed and Scopus using key words such as ART, drug discovery, adverse drug reaction and were conducted in October 2015. There was no exception made to limit inclusion of relevant clinical trials and the trials referenced were published between February 2004 and October 2016. However, clinical justification for the use of this study in treatment of ART and its ADR were selected from physicians, scientists, clinical pharmacist and it was 200 patients sample $(1,2)$.

\section{Inclusion criteria}

Patients with aging above 19 years. Patients having previous history of medical, medication problems in ART. The patients who are willing to take part in the study without any hesitation. Patients who are not willing to participate in the study without any hesitation.

\section{Study pattern}

Most scrutinized clinical study was collected from PubMed and Scopus $(3,4)$ This database has been curetted using parallel and unparalleled result for all most all type of adverse drug reaction. It has therefore necessary to identify those drugs for demonstration of compliance. This new clinical study of adverse drug reaction to ART can produce certain drug discovery and excitement of this pattern has that drug failed in their proposed activities due to resistance and negative balance in their adverse drug reaction best things to use the available clinical resources and recycle them. So it has consistent drug development and single max variable result towards ART with combinations will make actual drug discovery $(5,6)$.

\section{Age wise distribution of male population}

In this study total of 200 patients were enrolled, we have not seen death in the study. The males' population is 110 . The age wise male patients population ranges from 4 (7.27) patients were in the age group of 20-30 years, 54 (98.18) patients were in the age group of 30-40 years, 24 (43.63) patients were in the age group of 40-50 years, 18 (32.72) patients were in the age group of 50-60 years, 10 (18.18) patients were in the age group of $60-70$ years $(7,8)$ (Figure 1 and Table 1).

Age wise distribution of female population

In this study total of 200 patients were enrolled in the study. The Females population is 90 . The age wise Female patients population ranges from the 0 patients were in the age group of 20-30 and 32 (71.11) were from $30-40$ years, 38 (84.44) patients were in the age group of 40-50 years and 10 (22.22) patients were in the age group of 50-60 years and 6 (13.32) patients were in the age group of 60-70 years, 4 (8.88) patients were in the age group of 70-80 years, 0 patients were in the age group of $80-90$ years $(9,10)$ (Figure 2 and Table 1$)$.

${ }^{1}$ Department of Quality Assurance, Rajgad Dnyanpeeth's College of Pharmacy Bhor, India

Correspondence: Dr. Rahul Hajare,, Rajgad Dnyanpeeth's College of Pharmacy Bhor, India Telephone 91-9765704048, e-mail rahulhajare@rediffmail.com Received: April 20, 2017, Accepted: June 20, 2017, Published: June 27, 2017 
TABLE 1

Age wise distribution of male $\&$ female patients

$\begin{array}{cccc}\text { Age in years } & \text { Male patients } & \text { Age in years } & \text { Female patients } \\ 20-30 & 4(7.27) & 20-30 & 0(0) \\ 30-40 & 54(98.18) & 30-40 & 32(71.11) \\ 40-50 & 24(43.63) & 40-50 & 38(84.44) \\ 50-60 & 18(32.72) & 50-60 & 10(22.22) \\ 60-70 & 10(18.18) & 60-70 & 6(13.32) \\ \text { Total } & 110(110) & 70-80 & 4(8.88) \\ & & \text { Total } & 90(90)\end{array}$

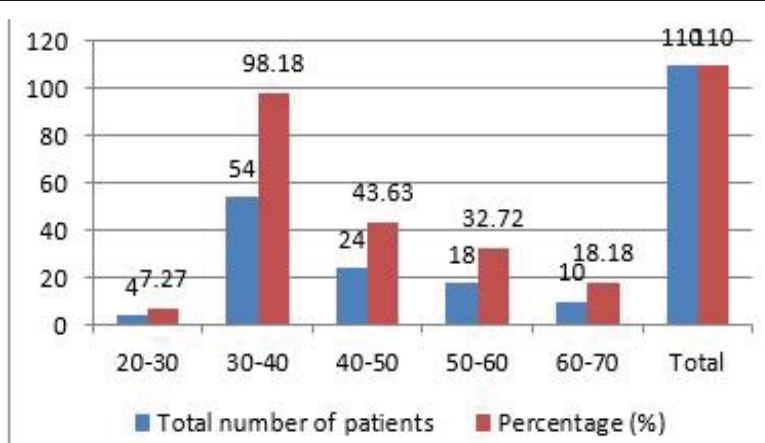

Figure 1) Age wise distribution of male patients showing percentage of distribution

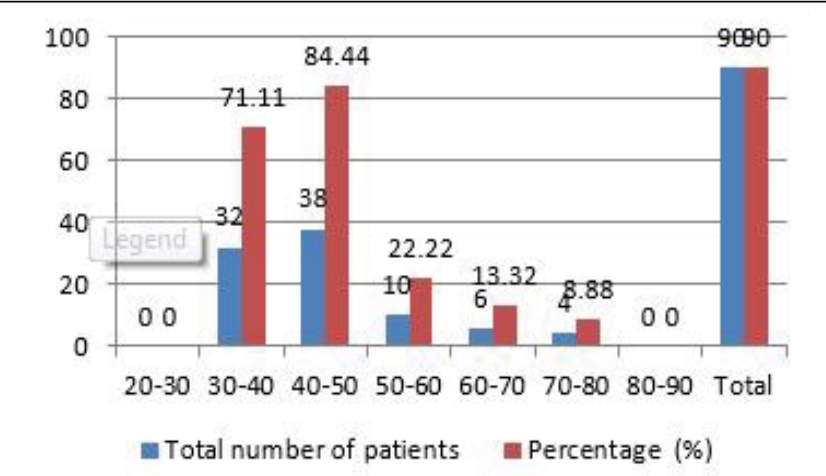

Figure 2) Age wise distribution of female patients showing percentage of distribution

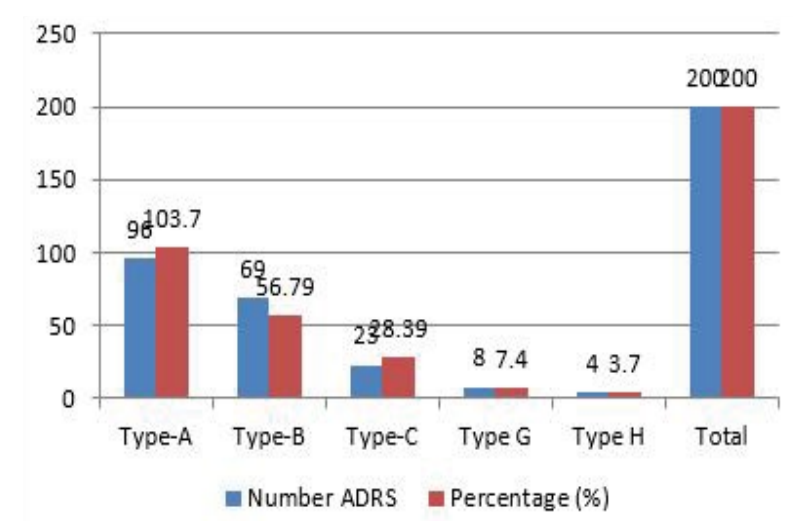

Figure 3) Types of ADRS and percentage of distribution

Type A-H: Type A: Augmented pharmacologic effects, Type B: Bizarre effects (or idiosyncratic), Type C: Chemical effects, Type D: Delayed effects, Type E: End-of-treatment effects, Type F: Failure of therapy, Type G: Drug suspected for ADR, Type H: Drug on past adverse event reported $(5,6)$.

\section{DISCUSSION}

Type A adverse effect has dose dependent and predictable, it can be alter limited point through molecular rearrangement which constitute approximately $80 \%$ of adverse drug reactions, are usually a consequence of the drug's primary pharmacological effect or a low therapeutic index of the
TABLE 2

\section{Showing medical diagnosis cases of HIV with other} comorbidities

\begin{tabular}{cc}
\hline Various medical diagnosis cases & Total \\
\hline HIV with hypertension and diabetes mellitus & 30 \\
HIV with pneumonia and urinary tract infection & 22 \\
HIV with tuberculosis & 32 \\
HIV with tuberculosis and leprosy & 22 \\
HIV with septicaemia & 14 \\
HIV with hypertension & 18 \\
HIV with gross anemia & 20 \\
HIV with agranulocytosis & 26 \\
HIV with asthma & 6 \\
HIV with other comorbidities & 10 \\
\hline Total Cases & 200 \\
\hline
\end{tabular}

TABLE 3

Types and number of adverse drug reactions

\begin{tabular}{ccc}
\hline Type of ADR & Number ADRS & Percentage (\%) \\
\hline Type-A & 96 & 103.7 \\
Type-B & 69 & 56.79 \\
Type-C & 23 & 28.39 \\
Type G & 8 & 7.4 \\
Type H & 4 & 3.7 \\
\hline Total & 200 & 200 \\
\hline
\end{tabular}

drug and they are therefore characterize by profiling in chemical structure $(11,12)$. They are dose-related and usually mild, although they may be serious or even fatal. Such reactions are usually due to when drug elimination is compromise and drug has not been efficiently identifying drug-disease interactions. Even through drug related problems were founded but after reporting information to physicians we are minimizing harmful to patients. In future clinical pharmacy services are one of the effective services in hospital to improve the quality of life of HIV patient's in the hospital. It is hoped that with the introduction of a new role, the situation for patient living with HIV AIDS in the hospitals in India will continue to improve and includes less relocation of PLHA (13) (Table 2). This also shows the outstanding importance of inpatient care in PLHA and provided indications that the patient's life could lead to less relocation, because relocation basically means risks and stress for all concerned adverse drug reaction. A comparison of the Type A, Type B, Type C. Type G, Type H characterized in this study with the earlier reported in Table 3 , types and number of adverse drug reactions forms suggested that all five characterized ADRS are novel clinical studies. We have also identified patients where previous history was positive for ADRS to similar drugs. So medication history plays a major role in preventing ADRS. Adequate previous history, when available can lead to avoidance of risky drugs and safeguard the lives of the patient. Hence, patients have to be encouraged to update knowledge with them and details of the drugs to which they are known or unknown to be ADRS and our study concluded that ART was the drugs that caused the maximum ADRS (Figure 3). So there is a need to be existential drug discovery to meet the right drug at right time to represents a single physical molecule of the compound to reduce the incidences of ADRs to the ART.

\section{CONCLUSION}

Other side of this study concludes clinically important ARV interactions are frequently observed in clinical practice, and often they can be anticipated by knowledge of the underlying mechanism. Whenever possible, these interactions should be prevented by avoiding the unnecessary (as evidence by ADR ART) use of poly-ART and by selecting co medications which are less likely to interact. If the use of potentially interacting drugs cannot be avoided, adverse clinical consequences may be minimized, as appropriate, by individualized dose adjustments guided by careful monitoring of clinical response and measurement of drug concentrations. There will be need to estimate the Etravirine and Rilpivirine levels through the method of analytical engineering that can resolve the status of the functional group lead adverse drug reaction.

\section{ACKNOWLEDGEMENT}

Renowned scientist Dr. R.S. Paranjape for data collection and guidance achieved for manuscript preparation. 


\section{REFERENCES}

1. Paranjape RS, Challacombe SJ. HIV/AIDS in India: an overview of the Indian epidemic. Oral Dis. 2016;22;1:10-14

2. Kalokhe AS, Stephenson R, Kelley ME, et al. Development and Validation of the Indian Family Violence and Control Scale. PLoS One 2016; 11 (1).

3. Paranjape RS, Thakar MR, Ghate MV, et al. Current status of research on HIV epidemic, pathogenesis, management and prevention in India Proc. Natl. Acad. Sci. Sect B. Biol. Sci. 2012 ;82 (1):167-180

4. Paranjape R, Ghate M, Kohli RM, et al. Coping strategies of HIV positive individuals and its correlation with quality of life in Pune, India. International Social Work 1-12. The Author (s) 2014; 817.

5. Ghate M, Tripathy S, Gangakhedkar R, et al. Use of first line antiretroviral therapy from a free ART programme clinic in Pune, India $\square$ A preliminary report. Indian J Med Res 2013;137:942-949.

6. Joglekar N, Paranjape R, Jain R, et al. Barriers to ART adherence \& follow ups among patients attending ART centres in Maharashtra, India. Indian J Med Res. 2011; 134 (6): 954-959.
7. Kohli RM, Sane S, Kumar K, et al. Modification of Medical Outcome Study (MOS) Instrument for quality of life assessment $\&$ its validation in HIV infected persons in India" Indian J Med Res. 2005;122:297-304.

8. Hajare R, Kulkarni S, Thakar M, et al. Technology development and design of novel 1, 3, 5-tri substituted-1h-indole-2, 3-dione Hiv-1 inhibitors with displays strategic nanomolar cytotoxicity. World J Pharm Pharm Sci. 2016; 5 (6): 391-397.

9. Rahul H, Smita K, Madhuri T, et al. Variability interaction between Etravirine and Rilpivirine: A retrospective. KJACT 2016; 2 (1):1-3.

10. Hajare RA, Kulkarni SS, Paranjape RS. Design space filling model, synthesis and evaluation of novel 2-indolinone HIV-1 inhibitors. International Journal of Advanced Research. 2015; 3 (12);1332-1335.

11. Gottlieb MS. Pneumocystis pneumonia-Los Angeles. MMWR Morb Mortal Weekly Rep. 1981;30 (21):250-252.

12. Nakagawa F. Projected life expectancy of people with HIV according to timing of diagnosis. AIDS. 2012;26 (3):335-343.

13. European Aids Clinical Society. Guidelines for the clinical management and treatment of HIV-infected adults in Europe, November 2014 Version 7.1. $2014 ; 24$ 